\title{
Sex and gender differences in nutrition research: considerations with the transgender and gender nonconforming population
}

\author{
Whitney Linsenmeyer ${ }^{1 *}$ (D) and Jennifer Waters ${ }^{2}$
}

\begin{abstract}
A sex- and gender-informed approach to study design, analysis and reporting has particular relevance to the transgender and gender nonconforming population (TGNC) where sex and gender identity differ. Notable research gaps persist related to dietary intake, validity and reliability of nutrition assessment methods, and nutrition interventions with TGNC populations. This is due in part to the conflation of sex and gender into one binary category (male or female) in many nutrition surveillance programs worldwide. Adoption of the Sex and Gender Equity In Research (SAGER) guidelines and the two-step method of querying sex and gender has the potential to exponentially increase the body of research related to TGNC health.
\end{abstract}

Keywords: Transgender, Gender nonconforming, Nutrition assessment

In this special issue on sex and gender differences in dietary intake and other dietary behaviors across the life course, we wish to bring to the table certain considerations regarding the transgender and gender nonconforming (TGNC) population.

Whereas sex refers to the biological attributes of females and males, gender refers to the socially constructed roles and behaviors associated with a feminine, masculine, or non-binary identity. The constructs of sex and gender are separate health determinants, and yet historically these variables have either been conflated or omitted entirely. Thus, a sex- and gender-informed approach to study design, analysis and reporting has great potential to increase the rigor and the relevance of scientific research [1].

\footnotetext{
* Correspondence: Whitney.linsenmeyer@health.slu.edu

'Doisy College of Health Sciences, Saint Louis University, 3437 Caroline St, St. Louis, MO 63104, USA

Full list of author information is available at the end of the article
}

This movement has particular relevance to the TGNC population where sex and gender identity differ. The term transgender may be used to describe a person whose gender identity differs from the sex that was assigned at birth, and the term gender nonconforming or genderqueer may be used to describe a person whose gender identity exists in a more fluid or multifaceted manner [2]. Worldwide estimates of TGNC demographics have been notoriously difficult to obtain given the variability in terminology, gender-based stigma and discrimination in many cultures, and respondents' concerns regarding confidentiality and anonymity; current estimates include $0.6 \%$ of the adult population in the United States, a figure that has doubled in the past decade $[3,4]$.

Nutrition-related considerations for the TGNC population are both clinical and psychosocial in nature. Those undergoing masculinizing or feminizing hormone therapy may experience weight gain, changes in body composition, altered lipid profiles, and changes in bone 
composition. Existing research also points to elevated rates of eating disorders, food insecurity, and discrimination both outside of and related to the medical community $[5,6]$. A recent scoping review identified notable research gaps related to dietary intake, validity and reliability of nutrition assessment methods, and nutrition interventions with TGNC populations [7].

Designing studies to address the identified research gaps may present significant challenges. Many largescale health surveillance databases frequently utilized by researchers to study the dietary behaviors and nutritional status of various groups of people are not as useful for studying the TGNC population due to the conflation of sex and gender into one binary category (male or female). This is observed in the United States with the National Health and Nutrition Examination Survey (NHANES) and the National Health Interview Survey (NHIS), as well as other nutrition surveillance programs throughout the world including the Canadian Health Survey (CHS), Australian Health Survey (AHS), and China Health and Nutrition Survey (CHNS).

The Sex and Gender Equity In Research (SAGER) guidelines offer researchers an approach to standardize reporting of sex and gender for research purposes. The guidelines endorse clear a distinction between sex and gender, as well as an analysis of sex and gender differences and similarities where appropriate [8]. When TGNC identities are included in this approach, commonly referred to as the two-step method, researchers have the opportunity to stratify data based on sex and a range of gender identities beyond male and female (Fig. 1) [9].

Adoption of this approach in nutrition surveillance programs and other systematic research could help to bridge the literature gaps on a range of topics impacting the TGNC population including dietary intake and

1. What is your gender identity? o Male

- Female

- Transgender man / Transman

- Transgender woman / Transwoman

- Genderqueer / Gender nonconforming

o Additional identity (fill in)

- Decline to state

\section{What sex were you assigned at birth? o Male \\ ○ Female \\ - Decline to state}

Fig. 1 Two-step method for the collection of sex and gender identity data eating behaviors, utilization of food and nutrition assistance programs, food access and security, weight history, engagement in physical activity, and consumption of dietary supplements. Ultimately, a sex- and gender-informed approach within systematic research has the potential to exponentially expand the body of research related to the health of the TGNC population.

\section{Abbreviations}

TGNC: Transgender and gender nonconforming; NHANES: National Health and Nutrition Examination Survey; NHIS: National Health Interview Survey; CHS: Canadian Health Survey; AHS: Australian Health Survey; CHNS: China Health and Nutrition Survey; SAGER: Sex and Gender Equity In Research

\section{Acknowledgements}

Not applicable.

Authors' contributions

WL and JW jointly outlined and drafted the submission. Both authors approved the final submission.

\section{Funding}

Not Applicable.

Availability of data and materials

Not Applicable.

Ethics approval and consent to participate

Not Applicable.

\section{Consent for publication}

Not Applicable.

\section{Competing interests}

The authors declare that they have no competing interests.

\section{Author details}

${ }^{1}$ Doisy College of Health Sciences, Saint Louis University, 3437 Caroline St, St. Louis, MO 63104, USA. ²Rush Copley Medical Center, 2000 Ogden Avenue, Aurora, IL 60504, USA.

Received: 15 December 2020 Accepted: 11 January 2021

Published online: 15 January 2021

References

1. Rich-Edwards JW, Kaiser UB, Chen GL, et al. Sex and gender differences research design for basic, clinical, and population studies: Essentials for investigators. Endocr Rev. 2018;39(4):424-39. doi:https://doi.org/10.1210/er. 2017-00246.

2. Center of Excellence for Transgender Health. Guidelines for the primary and gender-affirming care of transgender and gender nonbinary people, 2nd edition. https://transcare.ucsf.edu/guidelines Published June 17, 2016. Accessed November December 8, 2020.

3. Kritz B. The global transgender population and the international criminal court. Yale Hum Rts Dev LJ. 2014:17(1):1-38.

4. Flores AR, Herman JL, Gates GJ, Brown TN. The Williams Institute. How many adults identify as transgender in the United States? https://williamsinstitute. law.ucla.edu/wp-content/uploads/How-Many-Adults-Identify-asTransgender-in-the-United-States.pdf. Published June 2016. Accessed December 9, 2020.

5. Rahman R, Linsenmeyer W. Caring for transgender patients and clients: Nutrition-related clinical and psychosocial considerations. J Acad Nut Diet 2019;119(5):727-32.

6. Fergusson P, Greenspan N, Maitland L, Huberdeau R. Towards providing culturally aware nutritional care for transgender people: Key issues and considerations. Can J Diet Pract Res. 2018;79(2):74-9. doi:https://doi.org/10. 3148/cjdpr-2018-001. 
7. Rozga M, Linsenmeyer W, Cantell Wood J, et al. Hormone therapy, health outcomes and the role of nutrition in transgender individuals: A scoping review. Clin Nutr ESPN. 2020;40:42-56. https://doi.org/10.1016/j. clnesp.2020.08.011.

8. Heidari S, Thomas BF, De Castro P, et al. Sex and gender equity in research: Rationale for SAGER guidelines and recommended use. Res Integr Peer Rev, 1(2):1-9. https://doi.org/10.1186/s41073-016-0007-6.

9. Tate CC, Ledbetter IN, Youssef CP. A two-question method for assessing gender categories in the social and medical sciences. J Sex Res. 2013;50(8): 767-76. https://doi.org/10.1080/00224499.2012.690110.

\section{Publisher's Note}

Springer Nature remains neutral with regard to jurisdictional claims in published maps and institutional affiliations.

Ready to submit your research? Choose BMC and benefit from:

- fast, convenient online submission

- thorough peer review by experienced researchers in your field

- rapid publication on acceptance

- support for research data, including large and complex data types

- gold Open Access which fosters wider collaboration and increased citations

- maximum visibility for your research: over $100 \mathrm{M}$ website views per year

At $\mathrm{BMC}$, research is always in progress.

Learn more biomedcentral.com/submissions 